\title{
An effective strategy of real-time vision-based control for a Stewart platform
}

\author{
Josep M. Rossell \\ Department of Mathematics \\ Universitat Politècnica de Catalunya (UPC)
}

Avda. Bases de Manresa 61-73, 08242, Manresa, Spain

josep.maria.rossell@upc.edu

Josep Rubió-Massegú

Department of Mathematics

Universitat Politècnica de Catalunya (UPC)

Avda. Bases de Manresa 61-73, 08242, Manresa, Spain

josep.rubio@upc.edu

\author{
Jesús Vicente-Rodrigo \\ Department of EMIT \\ Universitat Politècnica de Catalunya (UPC) \\ Avda. Bases de Manresa 61-73, 08242, Manresa, Spain \\ jesus.vicente@upc.edu
}

\author{
Víctor Barcons \\ Department of EMIT \\ Universitat Politècnica de Catalunya (UPC) \\ Avda. Bases de Manresa 61-73, 08242, Manresa, Spain \\ victor.barcons@upc.edu
}

\begin{abstract}
A Stewart platform is a kind of parallel robot which can be used for a wide variety of technological and industrial applications. In this paper, a Stewart platform designed and assembled at the Universitat Politècnica de Catalunya (UPC) by our research group is presented. The main objective is to overcome the enormous difficulties that arise when a realtime vision-based control of a fast moving object placed on these mechanisms is required. In addition, a description of its geometric characteristics, the calibration process, together with an illustrative experiment to demonstrate the good behavior of the platform is given.
\end{abstract}

Index Terms-Stewart platform; parallel robots; inverse kinematics; vision-based control; tracking control.

\section{INTRODUCTION}

Essentially, a Stewart platform can be considered as a mechatronic system that involves different disciplines, such as electronics, mechanics, computing and control. It is a six degree-of-freedom mechanism composed by two rigid plates, i.e., a base plate and a movable top plate, connected by six extensible actuation legs.

The first platform was created in 1965 by D. Stewart as a full flight simulator [1]. In recent years, improved platforms have been used in industry areas such as automotive, mechanics, energy generation, aeronautic or civil engineering. Some applications are found in machine tool technology [2], high precision laser cutting [3], medical imaging [4], surgical operations [5], throwing platform of missiles, radio telescopes [6] or vehicle suspensions [7], just to name a few. Other recent applications can also be found in marine floating structures supporting precision instrumentation systems, cranes or wind turbines [8].

Our research team at the UPC has designed and built an electromechanic platform. Since this parallel robot is made up of a large number of components, such as wedges, universal joints, mechanical unions, aluminium plates, as well as small imperfections at the time of assembly, getting precise movements is really difficult. An important drawback in these mechanisms is their nonlinear character. Complex dynamics and kinematics often lead to introduce simplifications in the model. Frequently, a model linearization is a common strategy that facilitates the controller design and implementation but, at the same time, it may produce undesirable inaccuracies, mainly when the platform moves far from the linearization point.

Undoubtedly, one of the main goals of a Stewart platform is to generate prescribed multidirectional movements and rotations or to control an object placed on the top plate [9]. The leg lengths are variable and they can be controlled separately to perform the motion of the movable plate. To change the pose of the top plate relative to the base, the legs have to move in a coordinated direction to produce the desired displacement. In our implementation, the leg lengths are controlled by means of a motion control based on a CompactRio, a National Instruments device. To do this with high precision, a pre-calibration process is needed to establish the optimal parameters of the platform, that is, the attachment points at the base and at the top plate together with the leg length offsets, in order to minimize the leg lengths errors [10], [11].

The article focuses attention on setting up a smart camera to be used as positioning sensor of an object moving on the top plate to be controlled in real time. A large variety of problems appear when working with vision-based control: image distortions, delays in image capture, delays in image processing and actuators, precision in the location of an object, accuracy of the actuators response, etc. For real-time operations, it is crucial to select efficient methods avoiding time-consuming computation. LabVIEW has been chosen as the software to perform the movements and the control of the platform. It is a well-known graphical programming environmental for developing flexible and scalable applications.

In order to appreciate the accuracy of the platform, a balland-plate experiment is carried out. In this case, the platform 
has to drive a ball following a given circle, by using a smart camera as positioning sensor of the ball. The desired trajectory is compared with the actual trajectory to measure the tracking error. Obviously, different kinds of controllers could be implemented, but in this paper a proportional-derivative control is proposed. In future works, other controllers will be studied and tested.

The paper is organized as follows: Section II presents a detailed scheme of a Stewart platform. Section III is devoted to supply a basic background about the inverse kinematics. In Section IV, the calibration process of the platform prototype is briefly discussed. In Section V, the vision-based control strategy is presented. In Section VI, the experimental results are provided. Finally, the conclusions are given in Section VII.

\section{STEWART PLATFORM MECHANISM}

A picture of the platform, in fact a Gough-Stewart platform, built by our research team, can be seen in Fig. 1 .

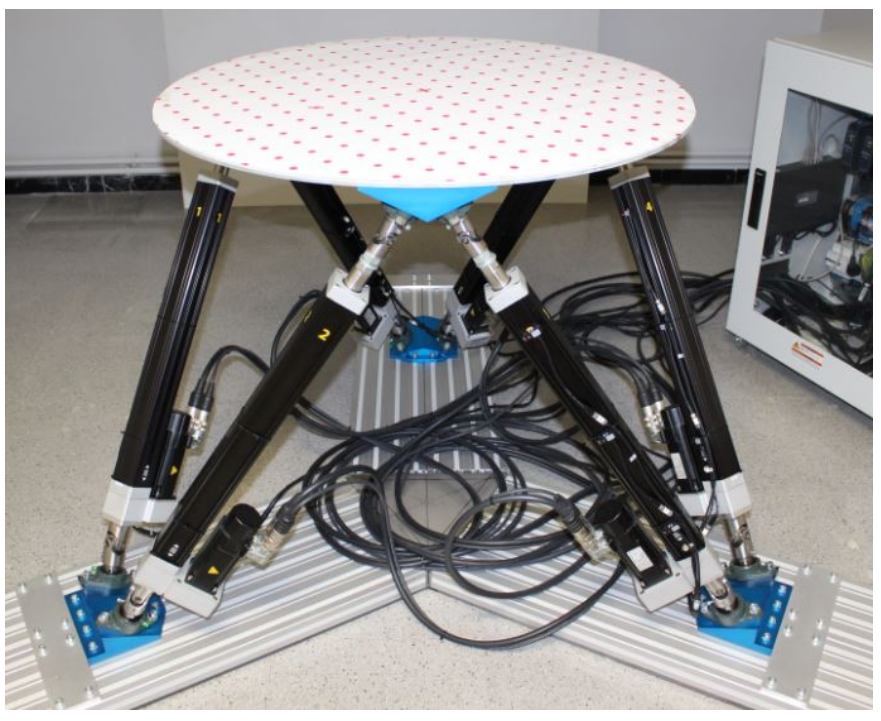

Fig. 1. Gough-Stewart platform at the UPC

To better understand the behavior of this kind of platforms, a simple scheme is depicted in Fig. 2. The leg lengths are variable and they have to be controlled separately to perform the motion of the top plate. Each leg is attached at the base and at the movable plate by universal joints. Since the rods of the legs are allowed to rotate about their axes, the universal joints act as spherical joints.

Denote by $O=[0,0,0]$ the origin of the base system and by $\{B\}=\{O ; x, y, z\}$ and $\{P\}=\left\{O^{\prime} ; x^{\prime}, y^{\prime}, z^{\prime}\right\}$ the reference systems of the base and the top plate, respectively. Let $b_{i}$ and $p_{i}, i=1,2, \ldots, 6$, the attachment points of the legs at the base and the top plate, respectively (see Fig. 3). The angles between the attachment points at the base are

$$
\begin{aligned}
& \alpha_{i}=\frac{\pi}{3}(i-1)-\frac{\theta_{B}}{2}, \text { for } i=1,3,5, \\
& \alpha_{i}=\alpha_{i-1}+\theta_{B}, \text { for } i=2,4,6
\end{aligned}
$$

and

$$
\begin{aligned}
& \beta_{i}=\frac{\pi}{3} i-\frac{\theta_{P}}{2}, \text { for } i=1,3,5, \\
& \beta_{i}=\beta_{i-1}+\theta_{P}, \text { for } i=2,4,6
\end{aligned}
$$

for the top plate.

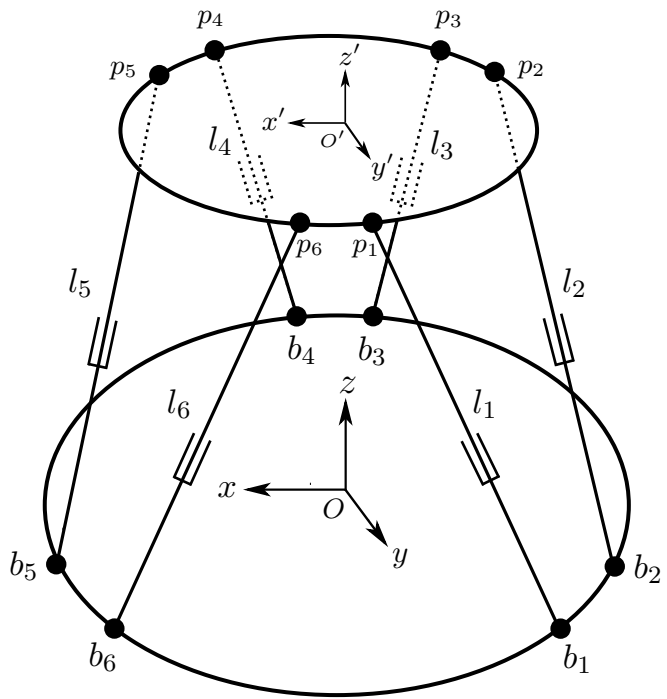

Fig. 2. Scheme of a Stewart platform

The location points $b_{i}$, with respect to the reference system $\{B\}$, can be described by the following vectors:

$$
b_{i}=\left[b_{i x}, b_{i y}, b_{i z}\right]^{T}=\left[r_{B} \cos \alpha_{i}, r_{B} \sin \alpha_{i}, 0\right]^{T}, i=1, \ldots, 6,
$$

where $r_{B}$ is the radius of the base. The location points $p_{i}$, with respect to the reference system $\{P\}$, are given by

$$
p_{i}=\left[p_{i x}, p_{i y}, p_{i z}\right]^{T}=\left[r_{P} \cos \beta_{i}, r_{P} \sin \beta_{i}, 0\right]^{T}, i=1, \ldots, 6,
$$

where $r_{P}$ is the radius of the top plate.

\section{INVERSE KINEMATICS}

A Stewart platform has a high nonlinear character and kinematic constraints appear. For this reason, forward kinematics is not a good methodology because it requires the solution of many nonlinear equations, mainly when a real-time control is needed. In addition, the forward kinematic problem has generally more than one solution.

The inverse kinematics scheme allows us to know the actuator variables $l(t)=\left[l_{1}(t), \ldots, l_{6}(t)\right]^{T}$, where the vector $l(t)$ is composed by the six leg lengths, in terms of the position and orientation of the top plate, at any instant $t$. Looking at Fig. 4, and by construction, we have

$$
l_{i}(t)=\left\|p(t)+R(t) p_{i}-b_{i}\right\|,
$$

where $b_{i}$ and $p_{i}$ are the attachment points given in (3) and (4), respectively, the vector $p(t)=\overrightarrow{O O^{\prime}(t)}=[x, y, z]^{T}$ contains the coordinates of the origin of the frame $\{P\}$ with respect to the frame $\{B\}$, and $R(t)$ is a rotation matrix that specifies the orientation of frame $\{P\}$ with respect to the frame $\{B\}$. 

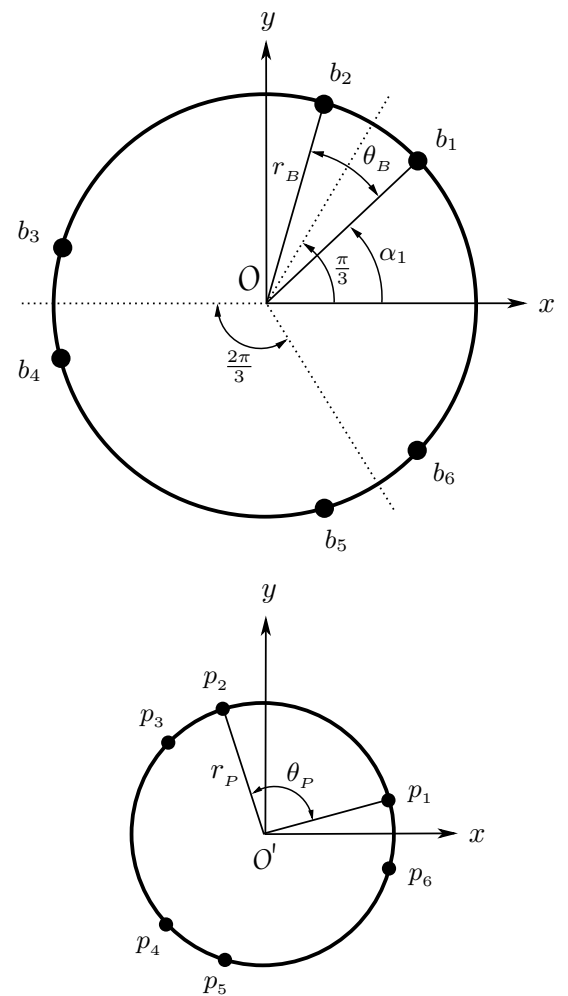

Fig. 3. Attachment positions at the base and the top plate

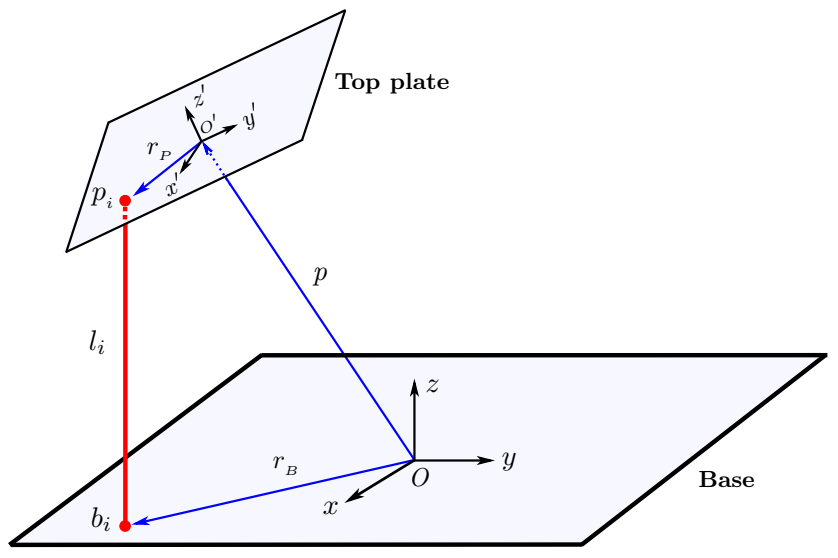

Fig. 4. Inverse kinematics scheme

The matrix $R=R(t)$ depends on the choice of the rotation axes sequence and, in this study, it is established as

$$
R=R_{z}(\alpha) R_{y}(\beta) R_{x}(\gamma)=\left[\begin{array}{lll}
r_{11} & r_{12} & r_{13} \\
r_{21} & r_{22} & r_{23} \\
r_{31} & r_{32} & r_{33}
\end{array}\right]
$$

with

$$
\begin{aligned}
& R_{x}(\gamma)=\left[\begin{array}{ccr}
1 & 0 & 0 \\
0 & c_{\gamma} & -s_{\gamma} \\
0 & s_{\gamma} & c_{\gamma}
\end{array}\right], R_{y}(\beta)=\left[\begin{array}{rrr}
c_{\beta} & 0 & s_{\beta} \\
0 & 1 & 0 \\
-s_{\beta} & 0 & c_{\beta}
\end{array}\right], \\
& R_{z}(\alpha)=\left[\begin{array}{ccc}
c_{\alpha} & -s_{\alpha} & 0 \\
s_{\alpha} & c_{\alpha} & 0 \\
0 & 0 & 1
\end{array}\right],
\end{aligned}
$$

where $s_{\alpha}=\sin \alpha, \quad s_{\beta}=\sin \beta, \quad s_{\gamma}=\sin \gamma, \quad c_{\alpha}=\cos \alpha, \quad c_{\beta}=\cos \beta$, $c_{\gamma}=\cos \gamma$. The matrix $R_{x}(\gamma)$ represents a rotation of $\gamma$ radians about $x$-axis; $R_{y}(\beta)$ a rotation of $\beta$ radians about $y$ axis; and $R_{z}(\alpha)$ a rotation of $\alpha$ radians about $z$-axis, with $\alpha, \gamma \in(-\pi, \pi)$ and $\beta \in\left(-\frac{\pi}{2}, \frac{\pi}{2}\right)$.

\section{CAlibration PROCESS}

After performing the assembly of all components of the platform, a calibration process must be carried out. In this section, some practical aspects of the performed calibration procedure are briefly discussed. Although a large variety of calibration methods can be found in the literature, there is no device that allows to measure the position and the orientation of the top plate with absolute precision. For instance, in [12], a stereo vision to measure the pose of a Stewart platform in real time is used.

For the acquisition of a complete location information of the top plate platform, an external sensor known as Coordinate Measuring Machine (CMM), has been used (see Fig. 5). The objective is to estimate the geometric parameters of the kinematic model, corresponding to six attachment points at the base $b_{i}=\left(b_{i x}, b_{i y}, b_{i z}\right)$, for $i=1, \ldots, 6$; six attachment points at the top plate, $p_{i}=\left(p_{i x}, p_{i y}, p_{i z}\right)$, for $i=1, \ldots, 6$, as well as six leg length offsets. Therefore, 42 real parameters have to be estimated [10], [11]. Initially, three target points are situated

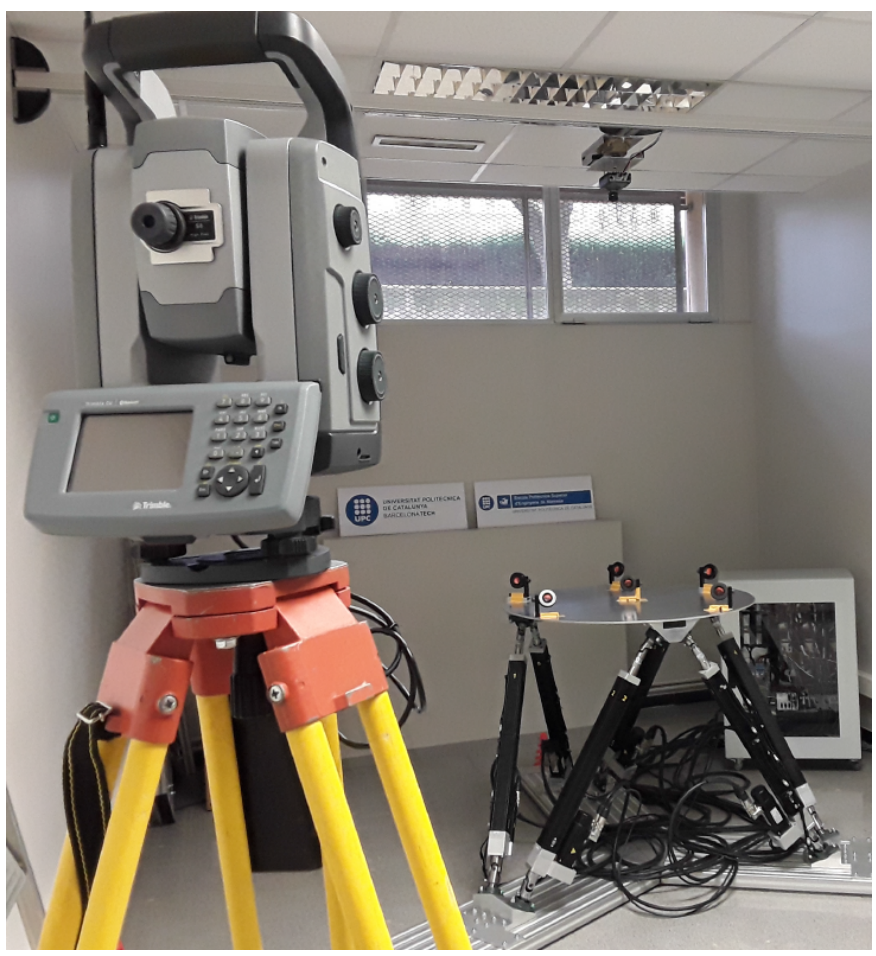

Fig. 5. Calibration process by using an electronic theodolite

at the base of the platform, defining its coordinate system. By means of an electronic theodolite, the positions of these three points are obtained and then transformed into the base coordinate system. Following a similar strategy, six target points are installed on the top plate, ensuring that at least three 
of them could be available to be measured by the theodolite at every calibration configuration.

After that, the top plate is moved to different poses, preferably extreme positions, together with other random positions. For each pose, the leg lengths given by the leg sensors are recorded. Moreover, ten measurements of three target points are provided by the theodolite and its average is considered as the true value of the corresponding measured point. In our calibration, 34 different poses of the top plate have been collected. With this information, and using a standard Levenberg-Marquardt least-square optimization algorithm, it is possible to establish the optimal parameter values in order to minimize the leg length errors.

Since this iterative algorithm needs an initial set of parameters, we start with the values obtained by direct measurement of the assembled platform, together with the parameter values provided by the technical specifications of the components. These initial geometric values and specifications are shown in Table I.

TABLE I

INITIAL GEOMETRIC PARAMETERS AND SPECIFICATIONS

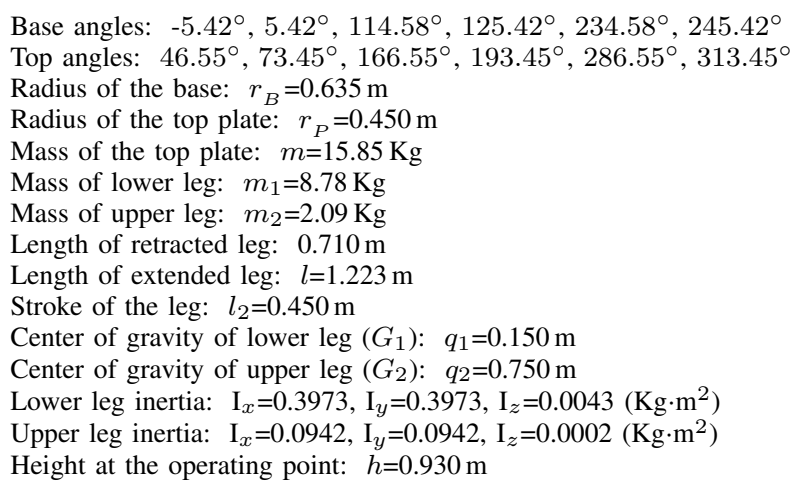

In Fig. 6, a scheme of an actuator leg with some of the parameters given in Table I can be observed.

\section{VISION-BASED CONTROL USING A SMART CAMERA}

\section{A. Selection of the camera}

First of all, it has been necessary to choose a smart camera capable of a quick and accurate detection of an object placed on the upper plate. The selected camera was a NI 1732 Smart Camera (VGA, Monochrome) which has total compatibility with LabVIEW software (see Fig. 7). It is a real-time device for artificial vision powered by a $400 \mathrm{MHz}$ PowerPC processor, that by combining the internal processor with a Charge-Coupled-Device (CCD) image sensor, provides a multifunctional vision system. The NI 1732 Smart Camera has a high capacity for analysis of images within the camera itself, which is crucial when working in real time.

\section{B. Camera set up}

The following sections describe how to create machine vision and image processing algorithms by using LabVIEW

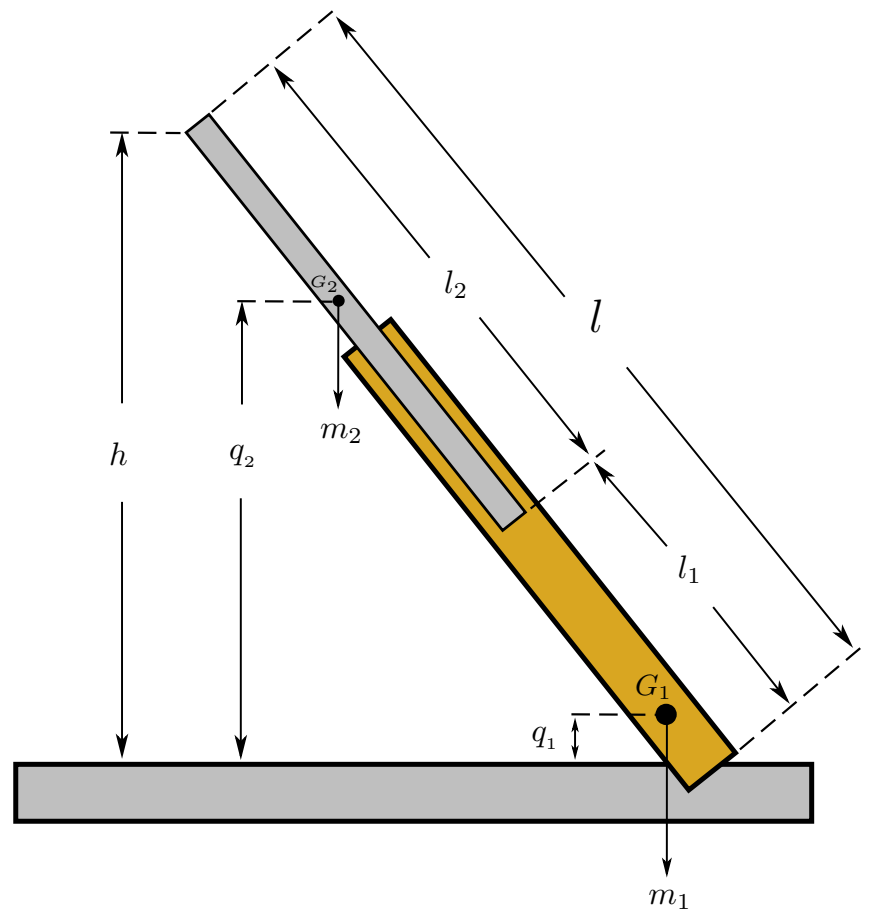

Fig. 6. Scheme of an actuator leg

Vision Assistant Module together with a Vision Real-Time Module [13].

In order to set up the camera, the following steps have been taken:

1) The selection of a lens having an appropriate field of view, by considering the distance between the camera and the top plate in its operating point or work position. The camera is installed on the vertical of the platform at $1050 \mathrm{~mm}$ from the working position and it has a resolution of $2.5 \mathrm{~mm}$.

2) The $x-y$ axes of the top plate are aligned with the $x-y$ axes of the camera.

3) A grid of red dots, with a uniform separation of $50 \mathrm{~mm}$, is set on the upper platform. This allows us to calibrate the camera in order to measure the real dimensions of any object situated on the top plate. Once the camera is calibrated, a red filter is installed in front of the lens so that the red dots do not affect the detection of any object.

4) The distortion of the image (fisheye lens distortion) when the platform moves away from the center is corrected.

5) The working area of the top surface is reduced from the physical top plate, $900 \mathrm{~mm}$ of diameter, to a circle of $760 \mathrm{~mm}$ due to the limited vision area of the camera when the platform moves up or to the sides.

\section{Position detection}

The main problem is the control of an object in real time, considering that there is a certain delay between the capture and processing of the image and the application of a control action. In our experimental setup, the object to be controlled is a ball of $22 \mathrm{~mm}$ of diameter. 


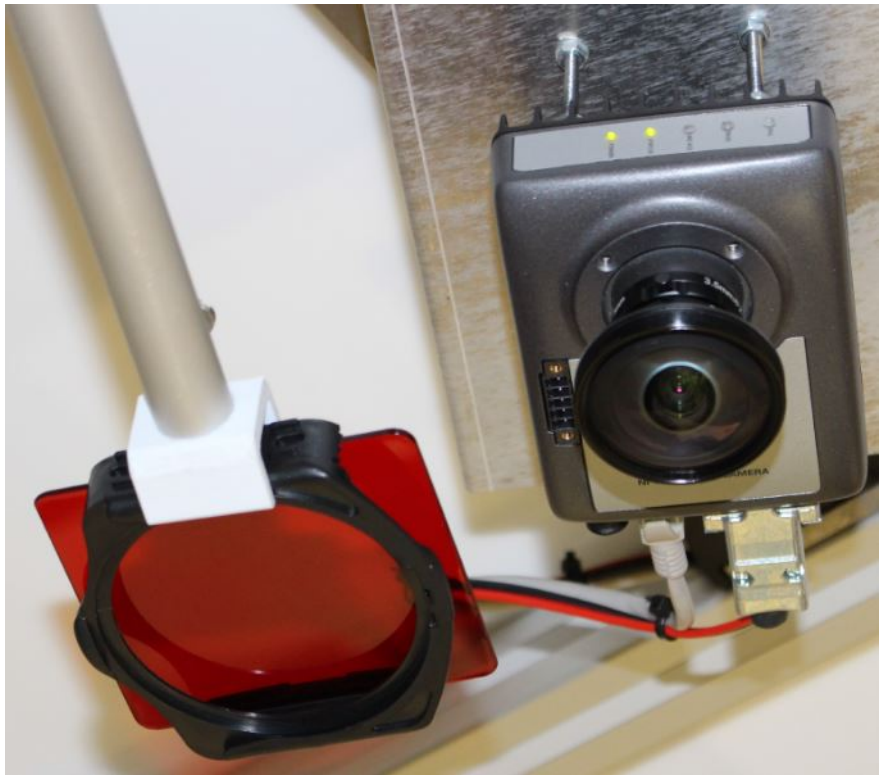

Fig. 7. NI 1732 Smart Camera with a red filter

To detect its position the following steps are taken:

1) A full scanning, following the $x-y$ axes directions, is applied to find the ball in the whole surface, that is, a circle of $760 \mathrm{~mm}$ of diameter.

2) The image of the ball is binarized to transform gray tones in black-white $(\mathrm{B} / \mathrm{W})$.

3) An approximate position of the ball is achieved in a B/W matrix using standard functions of the vision package software module.

4) In order to get an improved resolution, avoiding pixel problems near the binarization threshold, a small part of the gray image is used around the approximate position obtained in step 3). To locate the mass center of the ball a proper algorithm has been developed.

5) To work with a reduced scanning surface, the velocity of the ball is calculated and its position at the next instant is estimated. This strategy allows reducing the area where the camera will look for the ball to a small square of $250 \times 250 \mathrm{~mm}$.

6) Steps 2 to 5 are repeated while the ball is moving.

7) A rate of $20 \mathrm{~Hz}(50 \mathrm{~ms})$ of the ball position with a delay of $28 \mathrm{~ms}$ is achieved.

\section{A PD control}

A proportional-derivative (PD) control is applied to each axis $x, y$ by acting on the angles $\beta$ and $\gamma$ of the top plate, respectively. With the aim to compensate for the delay in image processing, i.e., the time between capturing and obtaining the position of the ball, the following recurrence for the $x$-axis is considered:

$$
\tilde{x}_{n}=(1-a) \tilde{x}_{n-1}+a \tilde{x}, \quad \tilde{x}_{0}=x_{0},
$$

where $n$ stands for the discrete instant time, $\tilde{x}_{n}$ is the desired position of the ball at the instant $n, \tilde{x}$ is the desired final position and $a$ is a parameter chosen by a trial and error method. The control action can be written in the following form:

$$
\beta_{n}=K_{p} e_{n}+K_{d} \dot{e}_{n}^{f},
$$

where $e_{n}=x_{n}-\tilde{x}_{n}$ is the error of the trajectory at the instant $n$, with $x_{n}$ the position given by the camera vision sensor at the instant $n$, and $\dot{e}_{n}^{f}$ is a filtered velocity error of the ball at the instant $n$, which can be computed as

$$
\dot{e}_{n}^{f}=\frac{e_{n}^{f}-e_{n-1}^{f}}{\Delta}
$$

with

$$
\Delta=t_{n}-t_{n-1}, \quad e_{n}^{f}=x_{n}^{f}-\tilde{x}_{n},
$$

and

$$
x_{n}^{f}=(1-b) x_{n-1}^{f}+b x_{n}, x_{0}^{f}=x_{0},
$$

where $b$ is a suitable parameter. In our case, $\Delta=5 \mathrm{~ms}, a=0.25$, $b=0.5$ and $K_{p}=K_{d}=0.03$. A similar recurrence as given in (8) is used for the $y$-axis.

\section{EXPERIMENTAL RESULTS}

The method presented in Subsection $D$ can be adapted to a general tracking problem with slight modifications. Previously, the platform has been calibrated taking into account the parameter values obtained after the calibration process detailed in Section IV.

In this section, a simple trajectory of a ball following a prescribed circle of $100 \mathrm{~mm}$ of radius is presented. Fig. 8 shows, in red, the ideal circular trajectory to be followed by the ball. Blue dots are the real positions of the ball when it follows the circular path. In Fig. 9, a zoom of a piece of

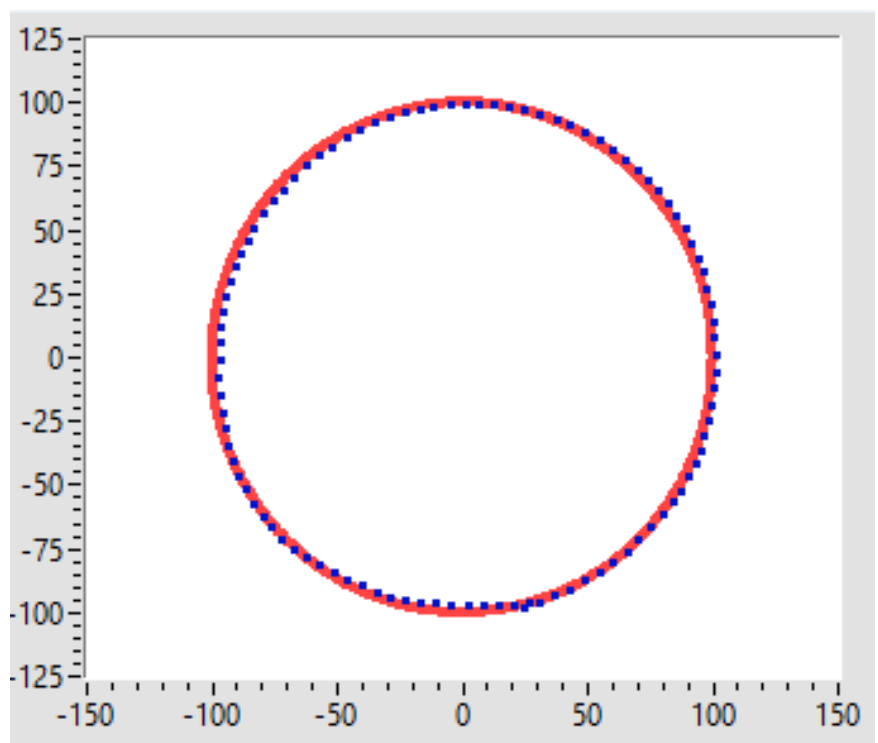

Fig. 8. Trajectory of the ball following a circle, in millimeters

trajectory is provided to observe the tracking error. Many of the positions practically match the ball over the predetermined circle. 


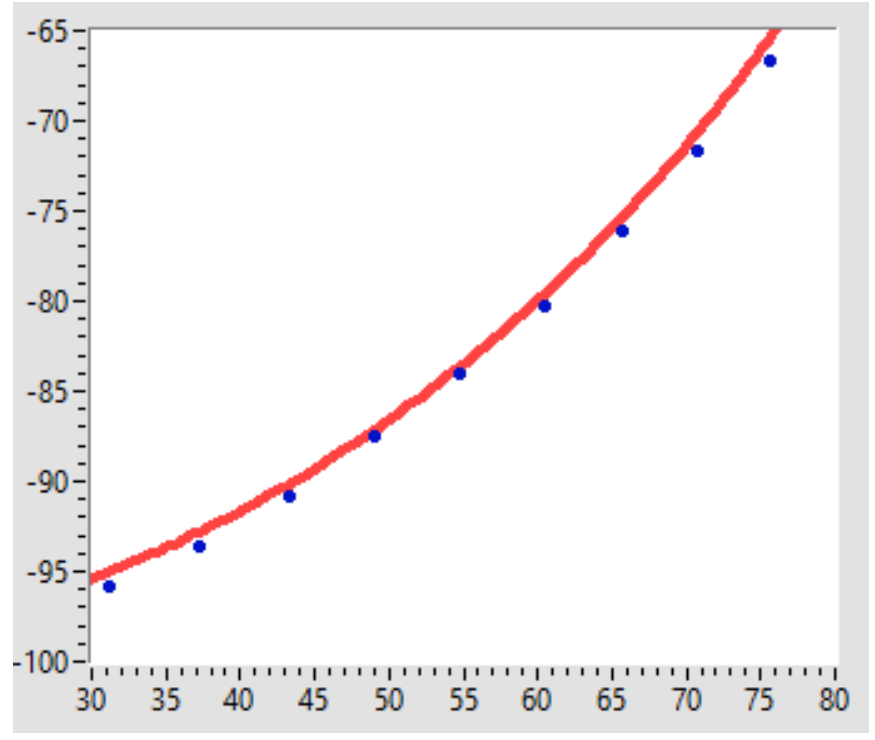

Fig. 9. Zoom of previous Fig. 8 circular trajectory

Finally, Fig. 10 shows the trajectory errors in a certain period of time. As it can be observed, the maximum error in absolute value is less than $3.8 \mathrm{~mm}$ along the path. High precision

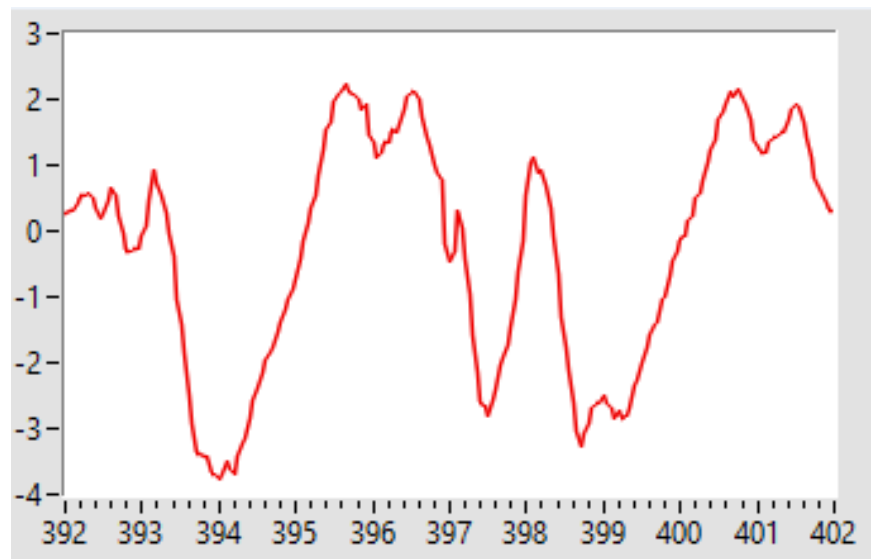

Fig. 10. Trajectory error (in millimeters) versus period of time (in milliseconds)

of movement is appreciated, mainly if we consider that the camera resolution is $2.5 \mathrm{~mm}$. These results demonstrate that the proposed control acts successfully and with high accuracy. A numerical simulation of the same platform by using Matlab can be found in [14].

\section{CONCLUSIONS}

This paper concentrates on a vision-based control for a Stewart platform designed and constructed at the laboratory of the UPC. First of all, the characteristics of this kind of mechanisms together with a basic background about the inverse kinematics have been presented. In addition, some practical aspects of the performed calibration procedure have been briefly discussed.
However, the main goal was to show the problems that arise when a real-time vision-based control is required. Due to some imperfections such as the assembly of the platform, delays in the capture of the images or delays in the image processing, the implementation of a real-time control is not an easy task. The proposed approach has produced positive results in a ball-and-plate experimental setup and can be useful in similar problems of practical interest. Other controllers will be studied and proposed in future works.

\section{ACKNOWLEDGMENTS}

This work was partially supported by the Spanish Ministry of Economy and Competitiveness under Grant DPI201564170-R/FEDER. We thank Jaume Martínez from ASOINDEL, S.L. for his help in using advanced tools of LabVIEW.

\section{REFERENCES}

[1] B. Dasgupta and T. Mruthyunjaya, "The Stewart platform manipulator: A review," Mechanism and Machine Theory, vol. 35, pp. 15-40, 2000.

[2] S. Pedrammehr, M. Mahboubkhah, and N. Khani, "A study on vibration of Stewart platform-based machine tool table," International Journal of Advanced Manufacturing Technology, vol. 65, no. 5-8, pp. 991-1007, 2013.

[3] L. Bruzzone, R. Molfino, and R. Razzoli, "Modelling and design of parallel robot for laser cutting applications," in Proceedings of the IASTED International Conference on Modelling, Identification and Control, Innsbruck, Austria, February 2002, pp. 518-522.

[4] S. Lessard, P. Bigras, I. Bonev, S. Briot, and V. Arakelian, "Optimum static balancing of the parallel robot for medical 3D-ultrasound imaging," in Proceedings of the 12th IFToMM World Congress, Besançon, France, June 2007.

[5] M. Wapler, V. Urban, T. Weisener, J. Stallkamp, M. Durr, and A. Hiller, "A Stewart platform for precision surgery," Transactions of the Institute of Measurement and Control, vol. 25, no. 4, pp. 329-334, 2003.

[6] J. Jáuregui, E. Hernández, M. Ceccarelli, C. López-Cajún, and A. García, "Kinematic calibration of precise 6-DOF Stewart platform-type positioning systems for radio telescope applications," Frontiers of Mechanical Engineering, vol. 8, no. 3, pp. 252-260, 2013.

[7] Y. Sam, N. Suib, and J. Osman, "Proportional integral sliding mode control for the half-car active suspension system with hydraulic actuator," in Proceedings of the 8th WSEAS International Conference on Robotics, Control and Manufacturing Technology, Hangzhou, China, April 2008, pp. 52-57.

[8] M. Horoub, M. Hassan, and M. Hawwa, "Workspace analysis of a Gough-Stewart type cable marine platform subjected to harmonic water waves," Mechanism and Machine Theory, vol. 120, pp. 314-325, 2018.

[9] B. Luo, W. Li, and H. Huang, "Experimental study of active vibration control for flexible beam by using a Stewart platform manipulator," in Proceedings of the 2011 IEEE International Conference on Mechatronics and Automation, Beijing, China, August 2011, pp. 1465-1470.

[10] Y. Hao, L. Changchun, L. Xiaodong, and Z. Jinying, "Calibration of Stewart platform based on coordinate measurement," in Proceedings of 2011 International Conference on Modelling, Identification and Control, Shangai, China, June 2011, pp. 469-474.

[11] H. Zhuang, J. Yan, and O. Masory, "Calibration of Stewart platforms and other parallel manipulators by minimizing inverse kinematic residuals," Journal of Robotics Systems, vol. 15, no. 7, pp. 395-405, 1998.

[12] A. Zuo, Q. Wu, and W. Gruver, "Stereo vision guided control of a Stewart platform," Proceedings of the 2002 IEEE International Symposium on Intelligent Control, 2002, pp. 125-130.

[13] N. Andreff, A. Marchadier, and P. Martinet, "Vision-based control of a Gough-Stewart parallel mechanism using legs observation," in Proceedings of the 2005 IEEE International Conference on Robotics and Automation, Barcelona, Spain, April 2005, pp. 2535-2539.

[14] J.M. Rossell, F. Palacios-Quiñonero, J. Rubió-Massegú, and J. VicenteRodrigo, "Tracking control for a Stewart platform prototype," in Proceedings of the International Conference on Advanced Mechatronics, Intelligent Manufacture, and Industrial Automation (ICAMIMIA), Surabaya, Indonesia, October 2015, pp. 58-63. 